\title{
The Components of Puzzle of Existential Fear: An Integrated Neuro-psycho-ecological Model of Understanding Experiences During the Covid-19 Pandemics
}

\section{Suela Ndoja*}

Clinical Psychologist-Division of Psychoclinical Service, Italian Albanian Association

"Progetto Speranza", Shkoder, Albania

*Corresponding Author: Suela Ndoja, Clinical Psychologist-Division of

Psychoclinical Service, Italian Albanian Association "Progetto Speranza”, Shkoder,

Albania.
Received: October 19, 2021

Published: January 07, 2022

(C) All rights are reserved by Suela Ndoja.

\section{Abstract}

The COVID-19 pandemic represents an extraordinary challenge to psychologists, social, public and health care institutions and policymakers. The paper outlines an integrated neuro-psycho-ecological model interpretation of such a puzzle of understanding experiences during pandemic. Our sense of self and others is threatened by the danger of i) being infected, ii) infecting other people, and (iii) the loss of social relation. This abnormal situation has an impact on us as subjectivities being intrinsically related with others and the world, leading to different neuronal and psychological responses based on our basic feelings, as that of fear. It is argued that fear experiences during the COVID-19 pandemic are organized on the neuro-psycho-ecological level around five interrelated dialectical domains, namely (1) fear system of circuits motivated to freeze and flee in apparent frights, (2) fear of infection per se, (3) fear of infecting the significant others/ fear for significant others, (4) fear of being infecting from the world/ of infecting the world, (5) fear of eco-system. These domains represent the neuronal, bodily, interpersonal and behavioral features of fear, respectively. Nowadays reading life because of daily confrontation with the death and connected existential fears is at the same time the threat and the value of this time. There are proposed some strategic points of addressing these fears and minimizing their impact by improving vital scenarios to live in a health way in our global village.

Keywords: Components of Puzzle; Existential Fear; Understanding Experiences; COVID-19; Coronavirus; Integrated Neuro-psychoecological Model

\section{Introduction}

On March 11, 2020, taking into account "alarming levels of prevalence and severity, and alarming levels of inaction," the World Health Organization characterized the situation of Severe Acute Respiratory Syndrome - Corona Virus 2 (SARS-CoV-2) as a pandemic [1]. The COVID-19 pandemic [2], is considered the invisible enemy, a ruthless invader that has infected the global psyche with fear and the bodies of the weakest people and the wider population with a potentially deadly disease. Therefore, this represents a unique time in our human history: it constitutes the place of global fear that is testing the boundaries of our traditions, cultures, and policies.
This knowledge, for us, as sensemakers of understanding the COVID-19 experiences, what we are really at great risk of because we ourselves are threatened by the coronavirus or someone we love is in great danger, has already created fundamental noise of concern and constitutes a constant concern. At the heart of all this noise [3], lies the element of one of the most fundamental and primary human emotions - existential fear because it characterizes transition periods, for which the initial state is known, but the duration and characteristics of transitions are unknown. For this, the COVID-19 pandemic poses a tremendous challenge to psychologists, socio-public, health care Institutions, and policymakers. On

Citation: Suela Ndoja. "The Components of Puzzle of Existential Fear: An Integrated Neuro-psycho-ecological Model of Understanding Experiences During the Covid-19 Pandemics". Acta Scientific Neurology 5.2 (2022): 11-22. 
the one hand, variable precautions, despite their severity, have resulted in and continue to significantly prevent the spread of the virus. On the other hand $[4,5]$, from a psychological point of view, such situations lead to feelings of insecurity, fear and despair. Apart from [6] are inextricably linked to feelings of helplessness and loss of a basic sense of security, financial stability and the ability to anticipate a brighter future.

Of course, the lack of emotional stability due to fear (as well as other negative emotions such as anxiety, stress, pain, etc.) can definitely give some advantages, such as providing information about our environment and our inner condition in interaction with the world around [7]. As the COVID-19 crisis challenges our social structure and social engagement system, we reflect on understanding how the crisis is affecting our mental and physical health, how we perceive the world and how we interact with others [8]. When we refer to the pandemic, it is essential to reinterpret and reformulate our reactions within an informed assessment of our nervous system recognizing that our reactions to the pandemic will make sense if informed by our sense of existence.

To give a clearer picture, this article aims to present an interpretation of the puzzle's components of existential fear according to an integrated neuro-psycho-ecological model of understanding the experiences during the COVID-19 pandemics. The article consists of three sections:

- $\quad$ The first section contains a description of the existential fear puzzle framework. The human mind has a powerful tool that aims to build feelings of self-reliance. Put it literally: to build the ability to form enduring narratives of our mental images for self-preservation. When self-sustainability is compromised, then the fear of existence creates its enigma within the life frame.

- The second section presents an interpretation scenario on integrated neuro-psycho-ecological model of understanding experiences during the Covid-19 pandemic. It is given that fear experiences during the COVID-19 pandemic are organized on the neuro-psycho-ecological level around five interrelated dialectical domains, namely (1) fear system of circuits motivated to freeze and flee in apparent frights, (2) fear of infection per se, (3) fear of infecting the significant others/ fear for significant others, (4) fear of being infecting from the world/of infecting the world, (5) fear of ecosystem. These areas represent the neuronal, bodily, interpersonal, and behav- ioral features of fear.

- The third section presents five conclusions. Nowadays reading life based on daily confrontations with death and the existential fear associated is even with the threat and value of this time.

- The fourth section presents some recommendations for addressing these fears and minimizing their impact by improving some strategic points of addressing these fears and minimizing their impact by improving vital scenarios to live in a health way in our global village.

\section{Section I: The existential fear puzzle framework}

Fear is widely regarded as an emotion with obvious functional and evolutionary significance [9]. It includes a combination of subjective disabilities (I am unable to cope with this), hopelessness (I can do nothing), helplessness (no one can help me), and catastrophizing (imminent death is approaching).

The human mind has a powerful tool that aims to build feelings of self-worth. When self-sustainability is compromised, then the fear of existence creates its enigma within the framework of life. In a word, fear is my feeling towards the cause of the damage to my existence, i.e., to put it better, the cause of the cessation of continuity and stability of oneself [10]. Some key psychoanalytic ideas are based on existential thinking, that is, existence before essence. Alternatively, existence is essence. Moreover, accordingly, the fear of death, and especially the fear of someone's death is the fundamental fear. Diseases (or agents that cause infection such as viruses and bacteria) pose major threats to the continuity and coherence of the self. Moreover, the psychoanalyst Sigmund Freud [11] detailed many sources of anxiety and fear, including the anxiety of annihilation - the fear of erasure. COVID-19 undoubtedly activates this anxiety [12], to the extent that it is present in humans, with the perhaps inevitable path to self-destruction and death.

This means that we as human beings tend to use the symbolism of our mind function to complete the stability of ourselves by relying more on our preconceived notions in terms of the Predictive Processing Model rather than on Actual Data [13,14].

More specifically, let us refer to the Pavlovian conditioning of fear, where averse learning is an experimental risk factor placed in the etiology and preservation of fear. The author, LeDoux [15] in his book "Emotional Brain" describes that the Pavlovian condition- 
ing of fear can be easily described in procedural terms. Therefore, a previous neutral stimulus being then paired at random with an unconditioned aversive stimulus that reflexively triggers an unconditioned response will alter the stimulatory functions of the nervous system. Thus, when presented alone, it functions as a conditioned stimulus that can trigger both conditional and autonomous conditioned emotional responses that we typically associate with frightening behaviors. For example, a year ago the word "Corona" was a neutral stimulus, at least in relation to fear as an emotional response. The words "virus", "disease", or "death" tend to be unconditional reactions that, when displayed, elicit an unconditional fear response and somatic emotional reactions [16]. Relating to this, operative conditioning considers fear as an emotional group and open behavioral responses are shaped and held by its consequences.

In this learning process, the likelihood of exhibiting a specific behavior increases or decreases according to the consequences that follow it. The main premise is that an environmental event or stimulus precedes a reaction, which is followed by a consequence that determines if the reaction occurs, more or less likely to occur again in the future [17].

In case of fear, a person may hear news about the number of deaths due to COVID-19 (antecedent stimulus), and release frightening reactions (crying, not sleeping, avoiding watching the news, etc.) followed by family members offering sedation (positive reinforcement) or reducing the level of fear (negative reinforcement), increasing the frequency of exposure to such fear reactions (especially those of avoidance and withdrawal) in the future [16].
In addition, some events cause frightening experiences due to the evolution of processes. The social spread of fear, for example, seems to be based in part on "primitive survival circuits" in the brain, for example, that will cause fear and avoidance at threatening signs, including other organisms showing fear [18]. In summary, all of the above data lead us to the integration of information that can be used to illuminate complex learning processes in times of crisis, throughout it and in the future, that can contribute, in whole or in part, to cognition, understanding of fear problems and treatment.

Section II: Integrated neuro-psycho-ecological model of understanding experiences during the Covid-19 pandemic

This COVID-19 situation poses a difficult challenge for psychologists, public, health care institutions, and policymakers because it brings together different conditions that are probably active together for the first time. For this, it is necessary to integrate different approaches to explain what has happened and what is happening in order to build the proper strategies and interventions to succeed.

Since events such as the COVID-19 pandemic cause fear due to the evolution of processes, it is organized on a neural, psychological, and ecological level. The neural level consists of the area of the nervous circuit system and the freeze-thaw motivation. The psychological level consists of interpersonal, bodily and behavioral aspects. The ecological level consists of aspects of the immune system and the physical environment. Furthermore, the dialectical structure of the identified fears may coexist with aspects of other common or converging fears among the domains. Consequently, the integrated neuro-psycho-ecological model is proposed, (Figure a) the description of which is given in next.

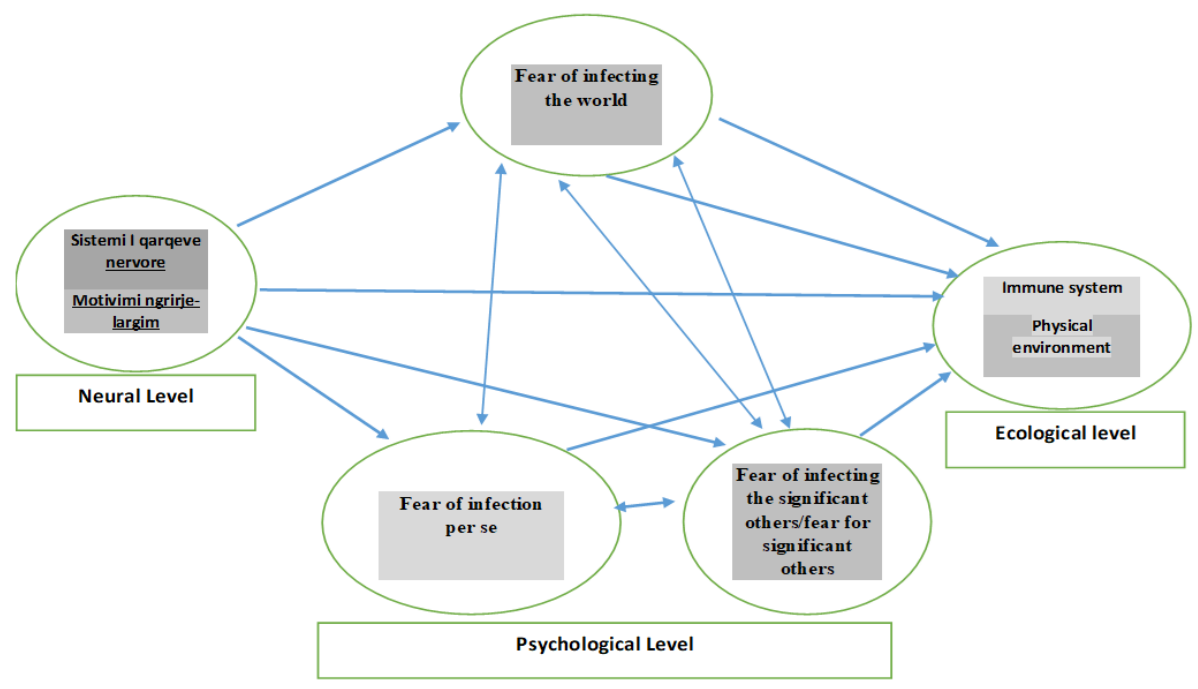

Figure a: Integrated neuro-psycho-ecological model. 
Neural level: To understand how threat alters both psychological and physiological processes, we need to understand and accept the 'a nervous system' model [8]. Functionally, the brain and visceral organs are connected by nerve pathways that send signals from the brain to our visceral organs and from the visceral organs to the brain. Thus, threat responses [8], through determinable and measurable pathways can have predictable effects on our mental and physical health. This integrated perspective of a nervous system encourages a better understanding of regulatory dynamics nervous system of an integrated nervous system. Next, let us ask the question: How exactly does the brain integrate information into its neuronal activity that it receives from the body's interoceptive data? Referring to the recently proposed theory of the spatio-temporal approach, which emphasizes the temporal structure of neural activity and its spatial patterns in the brain [19], it clarifies that the temporal structure of continuous neural activity represents the prediction of a priori (what will happen) that remain largely powerful for external information.

In this approach, interceptive input data represent a particular case. These inputs consistently provide critical information of survival value. Consequently, and unlike external inputs, interoceptive inputs also need to be continuously integrated with continuous spontaneous activity (resting state) [20]. Thus, a noisy interoceptive or afferent input can affect ongoing neural activity, and the neural circuits that receive this information may function abnormally. This, in turn, leads to somato-cardiac symptoms and abnormal interaction in anxiety disorders that may be closely related to abnormal interceptive processing of the brain.

System of motivated fear circles to freeze and move away from visible matches

The FEAR system is relational and neuro-ecological. This system [21-23] was created during brain evolution to help animals escape and to avoid the many dangers of the world. In humans, the highest levels of fear destroy the sense of security in the world by producing a higher level of stress and anxiety. In these extreme cases, the world is not perceived as a "safe haven".

The FEAR system can be activated by various external events as well as internal ones. These are usually triggered by specific external events that have been accompanied by pain or other threatening stimuli, but feelings of fear can also arise simply from the inter- nal dynamics of the brain, what is called the free-floating anxiety.

Indeed, when people are intensely stimulated, the circuits of the primary FEAR process motivate them to freeze and flee with apparent fear. In these conditions, people report being engulfed by anxiety and strong fear (e.g., "fear of death") without any necessary environmental cause [24]. Neuroscientists have tended to focus on information entering the FEAR system through so-called "high paths" (more cognitive inputs), and through "lower paths" (more primitive sensory inputs). The researcher, Panksepp, focused mainly on what he described as the "Royal Road", which is the very evolving FEAR system, a circuit that runs between the central amygdala and the gray periaqueductales of the midbrain, which directs the apparatus of instinctive action, which essentially helps animals avoid danger [25].

In addition, nerve studies show that the unconscious evaluation of a stimulus (say, the one that causes fear) begins before the stimulus is consciously processed. This is why, for example, our heart starts beating faster when we hear a knock on the window in the middle of the night, and only later, once we realize that the cause of the knock was a branch of a tree, the fear subsides. This is also probably, what is happening in our brain when we "tell ourselves" we need to maintain social distance and exercise caution. By doing so, we are less afraid. The amygdala appears to be the part of the brain that intervenes in the areas involved in the bodily expression of emotions and the areas of the brain that deal with conscious feelings.

\section{Psychological level}

What is happening nowadays with the spread of the COVID-19 pandemic is an existential threat to us as ourselves connected to others. Our sense of self and others is threatened by the risk of i) fear of infection per se, ii) fear of infecting the significant others/ fear for significant others and (iii) fear of infecting the world. This abnormal situation has an impact on us as subjectivities are inherently related to others and the world, leading to various neuronal and psychological responses based on our underlying feelings, such as the case of fear. Our selves are existentially threatened at its deepest level, namely its relationships with others and, more generally, with the world - this kind of existential fear for life. The emotional and behavioral response to the COVID-19 pandemic is multifactorial. It relies not only on external ingredients, but also on personal and innate ones as well. 
The Components of Puzzle of Existential Fear: An Integrated Neuro-psycho-ecological Model of Understanding Experiences During the Covid-19 Pandemics

\section{Fear of infection per se}

Throughout the evolutionary history, humans have been exposed to a wide and varying array of pathogens it has to do with the body and its signals [26]. In the context of the current COVID-19 pandemic, knowledge about the pandemic is limited and partial, which is deeply troubling. It happens that people do use the heuristic availability [27], which is, relying on information that can be recalled, usually information to which the person has recently been exposed. This leads to a one-sided reasoning, where; things that are remembered have a greater value to understand than those that cannot be readily remembered. Nevertheless, information is never \& neutral; and a careful balance between useful information and survival boosting and scary and paralyzing information can be difficult to achieve. For example, we may want to know how many people are infected in our local community [28]. While this type of information may help some people cope better, others may find it overwhelming and would prefer to be occult. What is most confusing, as with the other types of fear described in the context of the COVID-19 pandemic, is a rapid alternation of the need to know and the need to not know; such an alternation is likely to interfere with relevant decisions and actions. For example, searching for COVID-19 related information on the Internet can increase anxiety, worry, and thus lead to cyberchondria [29] and in the other hand makes one's believe that might suffer from similar physical symptoms. The body is the first organizer of our human experience [30] and "holds the result" [31] of events that threaten our physical and psychological integrity. In the current experience of the COVID-19 pandemic, fear of the body is associated with a sense of physical vulnerability due to which the body is a potential source of danger and cannot be trusted [29]. Such a perception of one's body is related to the fear of "betrayal of the body" through infection, which eventually leads to death. This fear of the body manifests itself in various ways in establishing the COVID-19 pandemic. People usually become overly vigilant about any bodily changes that COVID-19 infection may suggest, fearfully anticipating their surrender to it. The other body-related fear (fear of the body) has to do with the need to protect the body. Fear of the body and fear of the body can alternate very quickly, creating unstable behaviors and potentially harmful to the body. For example, we have sequentially heard from various national and international portals have reported that many people who survived a heart attack preferred to stay home rather than go to the hospital for fear of infection.

Fear of infecting the significant others/fear for significant others, relates to important interpersonal relationships, which are at the core of human identity, especially those involving related figures like parents, and people with whom we have close relationships. These individuals provide us with a safe haven and a secure base [32] from which we feel comfortable exploring our inner experience and the outside world. The COVID-19 pandemic has affected our perception of close interpersonal relationships with recommendations related to "social distancing". According to Relational Framework Theory [33], the way in which we verbally relate stimuli may be the source of much of the psychological suffering. This theory suggests that we learn to relate stimuli to our environment and that this connection may alter the psychological functions of those stimuli. The spread of coronavirus will keep everyone safe. The consequence of this is a perception that even loved ones can harm or kill us, albeit unintentionally. Therefore, instead of providing protection or a sense of security, a parent, child, or intimate partner becomes a potential threat, with our survival perhaps dependent on protecting ourselves from the people with whom we have the strongest affective ties. Conversely, we experience ourselves as potentially dangerous to our loved ones and responsible if infected. We are deprived of our normal role of caring for or protecting them. These changes have profound consequences not only in terms of how we relate to significant others, but also in terms of further damaging our sense of security and our need to "be there" for our loved ones.

\section{Fear of infecting the world}

It is related to the conscious self toward the world -. When the basis of our self in its underlying the world brain relation is disrupted or even lost, non-integration of internal and external stimuli might occur in order to avoid the painful experience of existential fear - this process might result in dissociation at different levels [34] terms "not-me" self-states. It relates to the consequences of fear behavior during the COVID-19 pandemic. Our lives also consist of actions, i.e. intentional and intentional activities that are not reflexive but have a subjective meaning [35]. As already noted, fears of infection per se, fears of infecting the significant others/fear for significant others and fear of infecting the world are domains often have a direct impact on our behavior. This is especially the case when fears; mutually opposite; alternates rapidly, producing indecision and paralyzing actions. For example, it can be very difficult to decide whether to go out because of the possibility of infecting the people passing by; such a person is divided between a duty to take care of others and the responsibility to keep them safe by avoiding such walking's out. At the same time, intero-exteroceptive disaggregation leads to disruption of the self's temporo-spatial 
alignment to the world [35]. If the world's exteroceptive stimuli can no longer be integrated with the body's interoceptive stimuli, the self becomes decupled and thus dissociated from the world. That ultimately leads to the collapse of inter-subjectivity and the self's neuro-ecological relation to its world that means a dissociation from self and the world.

\section{Ecological level}

Recent research in psychodynamic psychology and neurobiology suggests how the brain and self-construction depend on the interaction with the environment (e.g. important others) that plays a crucial role in developing self-awareness and connectivity with others [36,37]. The early growth and maturation of brain areas involved in personal and social development [38] is dependent on experience and requires nurturing other interactions with oneself in the context of connecting to the development of various related characteristics. With the construct of self, e.g., continuity, stability, regulation of cognitive and emotional states. This process of relational internalization is enabled by the human capacity for crosssubjectivity, adaptation, sensitivity and predisposition to create and associate common meanings, which are present from birth $[30,39,40]$.

Fear of the ecosystem- complex immuno-ecological interactions

The ecology of fear concerns the population-, community-, and ecosystem-level consequences of the behavioral interactions between predators and prey, i.e., the aggregate impacts of individual responses to life-threatening events. In this sense, the predator is COVID-19 and the prey is the human population. Darwin declared that organisms unable to adapt to the demands of their environment will fail to pass on their genes and consequently fall as casualties in the "war of nature" [41]. One of the most pervasive ecological demands is predatory avoidance. The relentless pressure to outwit predators while balancing homeostatic threats, such as resource depletion, has produced a nervous system that optimizes survival actions. These optimal actions provide the organism with a survival intelligence that permits appropriate responses to an array of environments and circumstances that range from non-threatening to life endangering. In humans, this behavioral repertoire is supported by a neurobiological system that has endowed us with a powerful set of intelligent survival mechanisms, promoting adaptation to changing ecologies and efficient navigation of natural dangers. Like the physiological immune system, the behavioral immune system includes both mechanisms of detection and response. When an external signal is detected that indicates the risk of infection (e.g. seeing another person with obvious manifestations of infectious disease), it triggers a cascade of emotional, cognitive, and behavioral responses that minimize the risk of infection (e.g. eg through the social avoidance of people who appear to pose a risk of infection). Therefore, in this case we are dealing with complex interactions: discovery-reaction and vice versa, which evolve depending on the context. Because relational frameworks can be arbitrarily based on context, the transformation of functions into relationships can have behavioral complications for people who are maladapted and not well adapted to the context $[42,43]$. For example, just as the Coronavirus began to receive all our attention as a foreign pathogen in the human body, so did talking about a traumatic event of the past or future (e.g., the death of a loved one from COVID-19) can bring feelings related to that event to the forefront for an individual. With a strong cultural message that undesirable psychological content is an obstacle to an effective life in natural environments in interaction with other social organisms. From this perspective, theory in ecology and evolution helps us to understand patterns and processes in natural systems and in human society. Based on ecological and evolutionary theory $[42,43]$, the indirect effects of pathogens that change the behavior of their hosts must be widespread in nature because we know that organisms - humans and many other species - usually change their behavior in response to pathogens - or as a result of infection, or to avoid infection.

Sometimes we have to face a kind of threat that proves to be completely out of control, sudden and difficult to find and prevent. It can even be immune to our biology (immune system) and chemical defenses (medicine), although it can live inside us just like a virus. Therefore, fear is easily associated with the worst horrifying memories we have experienced in our lives as individuals and as people [7].

Since our disease avoidance behavior occurs at individual and societal levels, not all of the observed dynamics fit into an ecological framework. For example, changes in policy and institutional support also affect in ways that are both positive and negative. Critically, while ecological theory can provide an insight into the avoidance of human disease and its consequences, it is important to recognize that behavior ours is complex, and the ways we interact with ecosystems are often unique to our species. 


\section{Section III: Conclusions}

This article aimed to present an interpretation of the puzzle's components of existential fear according to an integrated neuropsycho-ecological model of understanding the experiences during the COVID-19 pandemics. The descriptions obtained from the sections presented tell us considerably about the understanding of existential fear at the time of COVID19, about our experiences according to the integrated neuro-psycho-ecological model.

- First: It is clear that existential fear is a fixed emotional response triggered by the immediate danger that activates subcortical circuits by promoting feelings, thoughts, and behaviors that aim to maintain survival [7]. Such intimate connection of fear, self, and existence, is due to the way our brain and its spontaneous activity are organized, that is, it stabilizes itself by aligning to body and world in very much the same way the water stabilize its movements during its fall.

- $\quad$ Second: The threat of the coronavirus presents us with a double threat - collapse of our subjectivity as due to the risk of our body being infected and collapse of our inter-subjectivity as by social isolation of our self from others. The dual threat of both subjectivity and cross-subjectivity provides a deep dimension to our emotions including our fears and anxieties that result in existential fears [7].

- Third: A close connection of fear, of self and existence, owes to the way our brain organizes and its spontaneous activity, that is, it stabilizes by approaching the body and the world in the same way we stabilize our movements during movements adapting to the corresponding rhythm. The same approximation of our brain to the body and the world, that is, the temporo-spatial extension is threatened if not interrupted by the Coronavirus crisis and ultimately our world is threatened by both the virus and the protective measures of physical and social distancing [37].

- Fourth: At the deepest level of our existence, we are essentially connected to the other - we create ourselves through our inner connection to the other. The same inner connection with the other forms ourselves through what psychology describes as connection. The data [44] show that early life events like traumatic childhood events in our relationship with others strongly shape ourselves, leading to a connection dysfunction and major changes or deficits in the structure of the self that can be followed even into adulthood. Those traces are left in the form of a changed temporal-spatial dynamic in the spontaneous activity of the brain.

- $\quad$ Fifth: The spontaneous activity of our brain, although seemingly isolated within the skull, is essentially social and ecological, that is, neuro-social and neuro-ecological [7] - this is the lesson that brain image studies on ourselves and others, as psychological connection studies can teach us. Most importantly, it makes it clear that the social disruption caused by the Coronavirus urgency touches us so deeply on the basis of ourselves and its existence - it is no wonder that our selves can react no other than to existential fear, as who would not be afraid if the ground of someone in which he stands, is detached?

- $\quad$ Finally, we need full efforts not only from psychologists but also from other professionals to integrate a multidimensional approach within a larger framework to the benefit of the general state of health. We definitely need to take this step forward in order to face the possible consequences of this acute epidemic.

\section{Section IV: Suggestions}

Nowadays reading life based on daily confrontations with death and the existential fear associated is at the same time the threat and value of this time [37]. It should be borne in mind that referring to this paper, it is not about individualized therapeutic approaches [45], but about a modest simple ranking scenario that can be integrated to address these fears and minimize their impact as an response to experiences such as the ongoing outbreak of the COVID-19 pandemic.

\section{Scenario 1: Collective mentalizing fear}

Since COVID-19 is converted to a striking phenomenon pertaining to settings at all the levels of the ecological environment: within any culture or subculture, settings of a given kind-such as homes, streets, or offices-tend to be very much alike, whereas between cultures they are distinctly different, there is a great need to think of a collective reflective functioning. How can it be achieved? Mentalizing is synonymous with Reflective Functioning [46]. Mentalizing refer to the calm and deliberate task of trying to make sense of the mental states, beliefs, desires and fears that animate behavior in the self and others. The central importance of mentalizing to mental health arose out of attachment research with its core assumptions regarding the interpersonal nature of mind [47], and the belief that meaning in human life arises out of the groups we belong to, inti- 
mate pair-bonds that comprise families in all their diverse forms. In the protracted era of COVID-19, in order to achieve this, we need also to exercise having a psychological flexibility. It is about having a range of inter- and intra-personal skills that can be defined as the ability to "recognize and adapt to different situation requirements; repertoires change mentalities or behaviors when these strategies compromise personal or social functioning; maintain balance between important things in areas of life; and to be aware, open, and committed to behaviors that are consistent with deeply held values [48]. The terminology itself shows that this can be achieved by assessing the integration of all parts of the system. Thus, we all play a unique role to alter the situation. The principle applies not only to the developing person but to the others in one's world.

Scenario 2: Psychological resources: the defense mechanism and the practice of personal and social awareness

Finally, situations such as the COVID-19 pandemic can bring out both the best and the worst in humans. For this, people need to activate defense mechanisms that are healthy and that help them cope with the crisis of experiencing COVID whether from the news, from relatives or beyond. It is a test of human ability to empathize, to show solidarity and put the good of society above its own interests. Thus, individual responsibility is crucial at the time of the COVID-19 pandemic. Individuals are moral agents and their actions can positively or negatively affect other people's lives. Therefore, promoting pandemic awareness and responsible behavior toward oneself and others can help people feel morally supported when confronted with their fears.

After all, it is a responsible action that can be critical to our survival. It is also very important in these times to know and be aware of the semiotic capital [49] which is the set of meanings embodied and symbolic (implied habits, worldview, values, social representations, cognitive patterns, pockets of implicit and external knowledge) that promote the ability of individuals to internalize the collective dimension of life and, in doing so, to make a such an internal dimension a pre-reflective, embodied component of experience: a concrete fact, a meaningful subjective regulatory component of his/her identity.

In doing so, a seeker of the meaning of COVID-19 experiences can feel the relevant aspects of social and political life endowed with life value [50]. First, one may feel the collective interest as something that matters, the common good as a super-ordered framework of meaning that bases unforeseen attitudes and actions in concrete situations. Coincidentally, this view involves looking at psychosocial resources based on the cultural environment, and therefore seeing individual ways of feeling, thinking, and acting as manifestations of the level of access to semiotic capital [50].

\section{Scenario 3: Development of a semiotic cultural environment}

The observations in this scenario are intended to have more than a merely speculative nature. They suggest an interpretation of what could be the semiotic impact of COVID-19 on the cultural milieu. That is, the sign "pandemic" is able to denote very many aspects of the social landscape, and is therefore used within a great many discourses and social practices, with different cultural and psychosocial content - e.g., one finds "pandemic" and associated signs (e.g., "contagious", "COVID-19, "crisis") within expressions of fear, concern, anger against those in charge, as well as in expressions of national pride, scientific language, institutional engagement, practices of active citizenship, acts of solidarity, social supports, thankfulness, hope and so forth [51]. Relating to this, the core tenet of creating a semiotic cultural environment is that affective semiosis sets the sensemaker's mind so as to constrain one's identification with sets of beliefs and attitudes that are available in the shared cultural environment the sensemaker is living. In other words, the preferred pattern of affective meaning in terms of which the seeker of understanding shapes the experience operates as a filter that "tunes" into just a certain area of the cultural environment; in so doing, the preferred affective meaning constrains the chance of interiorizing only the cultural worldviews and beliefs that fit with it [51].

Scenario 4: Developing psychological interventions and projects

The observations in this scenario are focused on the development of secure relationships that has ascended to the development of facing the fear of others might be afraid to seek meaning. Safe attachment is associated with positive quality of interpersonal interactions [52] and me a capacity for effective and controlling self-regulation [53]. When problems arise in the context of fear of COVID-19 intervention is required in the clinic, the beginning of the capacity to enable and think about the inner condition and interpersonal motivations of others may be critical to growth [54] and do [55]. In addition, is it possible to assess the quality of the connection, identify problems in this work, and address any internal conflicts that may surround the problem [56]. The long-term consequences of prolonged distress can have disastrous effects on 
cognitive functioning, including damage to the memory and hippocampal neuroplasticity as well as an increased risk of developing symptoms [57]. For this reason, at the end of the fourth scenario of intervention, it should be possible for the professional community of psychologists to develop interventions and projects in order to stem the psychological consequences of enforced quarantine or difficult working environments in an emergency. For all the reasons reported herein, fine tuning effective psychological interventions are a future need, given that after the public health emergency psychological distress emergency seems at the horizon. Certain preventive actions are prompted by the fear of contracting the COVID-19 virus [58]. Clearly, it is also a time that favors taskoriented and problem-focused forms of coping in order to help people navigate the challenges of daily life. Of course, for those people who have been physically isolated and perhaps in quarantine, there is a need to find ways to psychologically distract oneself and avoid the types of rumination and worry that will not only add to distress, they will also undermine immune system functioning. However, when the pandemic has passed and we return to our "new normal" it is vital to have widespread implementation of programs that boost the resilience of people of all ages. Here we must underscore that resilience is essential from the cradle to the grave because our elders most certainly need to be resilient when they start to wonder how much they have mattered. The core focus of such resilience building programs should be enhancing the sense of self-worth that people have so that they are less likely when they in daily life and in crisis situations to have the experience of such fears as the fear of missing out and not keeping up with others, etc.

\section{Scenario 5: Advancing social and public health policies}

The final observations in this scenario closure refer to the key question to ask is what will happen once the pandemic is everlasting, when the peak of infection has been reached and the spread starts to slow down?

- $\quad$ First, from our individual, community and global perspective as has been recognized, the lockdown needs to be kept up for several weeks, even months after the peak but it cannot last so far.

- Second, once the pandemic has been stopped and zero new cases are observed, this does not mean that the virus will have been fully eradicated
- $\quad$ Thus, these tormenting thoughts later will make it necessary to manage the transition to normality, maintaining high levels of alert without falling into the temptation of a local shortterm strategy

- The transition scenario draws the attention of institutional efforts of trans-national coordination of socio-sanitary policies (e.g., in fields like scientific and pharmaceutical research, health organization, regulation of frontiers, transportation); to elaborate sustainable strategies. We are still in a clime of an ongoing covid era and there are seen that people in their daily habits (e.g., limitation of movements, use of protective masks, adoption of social caution; trackability of social contacts) are still following the initial measures to protect oneself the loved one and the surrounding world

- Third, the transition scenario can be expected to end only when a medical solution to COVID-19 is found, something experts are implementing vaccines and still foreseeing for a third one [59].

Hence, the awareness of the need to empower the health system, the recognition of the capacity of democratic institutions to cope with such a complex crisis; the perspective of a possible, more advanced balance between individual rights and systemic constraints, in the domain of the protection of strategic common goods like health; the role of scientific knowledge in political decision-making ore over, it would be highly desirable that institutions - from territorial to international level - could make an effort to learn from the experience and elaborate more or less radical solutions to avoid future pandemics.

\section{Conclusion}

In summary, the components that enable us as seekers COVID-19 experience to understand the systemic dimension of experience, and thus making the regulation of interdependence with others an essential component of ourselves, can be seen as a kind of "semiotic antibody" of the great prominence of the anxiety-like sensation that characterized the current socio-political scenario, or conspiracy theories before the pandemic uprising, and which risks being the cultural and subjective framework that shapes the psychosocial ways of crisis treatment. One can be optimistic that, also thanks to the societal reaction fuelled by the fear response, the reaction to the acute pandemic crisis will be able to produce results in the development of a lively and healthy macrosystem [60]. 


\section{Bibliography}

1. Bedford J., et al. "COVID-19: towards controlling of a pandemic". Lancet 395.10229 (2020): 1015-1018.

2. Polizzi C., et al. "Stress and Coping in the Time of COVID-19: Pathways to Resilience and Recovery". Clinical Neuropsychiatry 17.2 (2020): 59-62.

3. Graber JA and Brooks-Gunn J. "Transitions and turning points: Navigating the passage from childhood through adolescence". Developmental Psychology 32.4 (1996): 768.

4. Hawryluck L., et al. "SARS control and psychological effects of quarantine, Toronto, Canada". Emerging Infectious Diseases 10.7 (2004): 1206-1212.

5. Wang C., et al. "Immediate Psychological Responses and Associated Factors during the Initial Stage of the 2019 Coronavirus Disease (COVID-19) Epidemic among the General Population in China". International Journal of Environmental Research and Public Health 17.5 (2019).

6. Forner CC. "A Missing Ingredient in a Time of Fear: Carers are not the Bucket". Clinical Neuropsychiatry 17.2 (2020): 80-84.

7. Porcelli P. "Fear, anxiety and health-related consequences after the COVID-19 epidemic". Clinical Neuropsychiatry 17.2 (2020): $103-111$.

8. Porges SW. "The COVID-19 Pandemic is a paradoxical challenge to our nervous system: a Polyvagal Perspective". Clinical Neuropsychiatry 17.2 (2020): 135-138.

9. Presti G., et al. "The Dynamics of Fear at the Time of COVID-19: A Contextual Behavioral Science Perspective". Clinical Neuropsychiatry 17.2 (2020): 65-71.

10. Damasio A. "The Strange Order of Things. Life, Feeling, and the Making of Cultures". New York: Pantheon Books (2018).

11. Freud S. "Inhibitions, symptoms and anxiety. The Standard Edition of the Complete Psychological Works of Sigmund Freud. London: The Hogarth Press". according to researcher Steel et al 20 (1926): 87-156.

12. Steele H. "COVID-19, Fear and the Future: An Attachment Perspective”. Clinical Neuropsychiatry 17.2 (2020): 97-99.

13. Smith R., et al. "An Embodied Neurocomputational Framework for Organically Integrating Biopsychosocial Processes: An Application to the Role of Social Support in Health and Disease". Psychosomatic Medicine 81 (2019): 125-145.
14. Van den Bergh O., et al. "Symptoms and the Body: Taking the Inferential Leap". Neuroscience and Biobehavioral Reviews 74 (2017): 185-203.

15. LeDoux J. "The emotional brain". New York: Simon and Schuster (1996).

16. Presti G., et al. "The Dynamics of Fear at the Time of COVID-19: A Contextual Behavioral Science Perspective". Clinical Neuropsychiatry 17.2 (2020): 65-71.

17. Rescorla RA. "Associative relations in instrumental learning: The eighteenth Bartlett memorial lecture". The Quarterly Journal of Experimental Psychology 43.1 (1991): 1-23.

18. Mobbs D., et al. "The ecology of human fear: Survival optimization and the nervous system". Frontiers in Neuroscience 9 (2015): 55.

19. Northoff G. "Is the self a higher-order or fundamental function of the brain? The "basis model of self-specificity" and its encoding by the brain's spontaneous activity". Cognitive Neuroscience 7.1-4 (2016): 203-222.

20. Northoff G. "How Is Our Self Altered in Psychiatric Disorders? A Neurophenomenal Approach to Psychopathological Symptoms". Psychopathology 47.6 (2014): 365-376.

21. Panksepp J. "The psychoneurology of fear: Evolutionary perspectives and the role of animal models in understanding human anxiety". In G. D. Burroës, M. Roth, and R. Noyes Jr. (Eds.) Handbook of Anxiety (1990): 3-58.

22. Panksepp., et al. "The basic neuroscience of emotional experiences in mammals: The case of subcortical FEAR circuitry and implications for clinical anxiety". Applied Animal Behaviour Science 129.1 (2011): 1-17.

23. Davis M., et al. "Phasic vs Sustained Fear in Rats and Humans: Role of the Extended Amygdala in Fear vs Anxiety". Neuropsychopharmacology 35.1 (2009): 105-135.

24. Nashold BS., et al. Sensations Evoked by Stimulation in the Midbrain of Man". Journal of Neurosurgery 30.1 (1969): 14-24.

25. Panksepp J. "Textbook of Biological Psychiatry". Hoboken, NJ, United States: Wiley

26. Troisi A. "Fear of COVID-19: insights from evolutionary behavioral science”. Clinical Neuropsychiatry 17.2 (2004): 72-75. 
27. Tversky A and AMP Kahneman D. "Availability: A heuristic for judging frequency and probability". Cognitive Psychology 5.2 (1973): 207-232.

28. Schimmenti A., et al. "The four horsemen of fear: An integrated model of understanding fear experiences during the COVID-19 pandemic". Clinical Neuropsychiatry 17.2 (2020): 41-45.

29. Starcevic V. "Cyberchondria: Challenges of Problematic Online Searches for Health-Related Information". Psychotherapy and Psychosomatics 86.3 (2017): 129-133.

30. Stern DN. "The Interpersonal World of the Infant: A View from Psychoanalysis and Developmental Psychology". New York, NY: Basic Books (1985).

31. Van der Kolk B. "The Body Keeps the Score: Mind, Brain and Body in the Transformation of Trauma". New York, NY: Penguin Random House (2015).

32. Bowlby J. "A secure base. Clinical applications of attachment theory". London, UK: Routledge (1988).

33. Hayes SC., et al. "Relational Frame Theory: A Post-Skinnerian account of human language and cognition". New York: Plenum Press (2001).

34. Bromberg PM. "Standing in the spaces: Essays on clinical process trauma and dissociation". Routledge.

35. Davidson D. "Essay on Actions and Events". Oxford, UK: Oxford University Press (1980).

36. Schore AN. "Attachment and the regulation of the right brain". Attachment and Human Development 2.1 (2000): 23-47.

37. Scalabrini A., et al. "The self and its world: a neuro-ecological and temporo-spatial account of existential fear". Clinical Neuropsychiatry 17.2 (2020): 46-58.

38. Pfeifer JH and Peake SJ. "Self-development: Integrating cognitive, socioemotional, and neuroimaging perspectives". Developmental 2.1 (2012): 55-69.

39. Trevarthen C. "Intrinsic motives for companionship in understanding: their origin, development, and significance for infant mental health". Infant Mental Health Journal 22 (2001): 95-131.

40. Tronick E. "The Neurobehavioral and Social-emotional Development of Infants and Children". New York, NY, United States of America: W. W. Norton and Company (2007).
41. Darwin C. "The Descent of Man and Selection in Relation to Sex". London: John Murrey (1871).

42. Curtis VA. "Infection-avoidance behaviour in humans and other animals". Trends in Immunology 35.10 (2014): 457- 464.

43. Weinstein N. "Unrealistic optimism about our future life events". Journal of Personality and Social Psychology 39 (1980): 806-820.

44. Stern DN. "The Interpersonal World of The Infant". Amsterdam, Netherlands: Adfo Books (2000).

45. Gazzillo F., et al. "Effectiveness is the gold standard of clinical research". Research in Psychotherapy: Psychopathology, Process and Outcome (2017).

46. Steele $\mathrm{H}$ and Steele M. On the origins of reflective functioning. In F. Busch (Ed). "Mentalization: Theoretical considerations, research findings, and clinical implications". Psychoanalytic Inquiry Book Series 29 (2008): 133-158.

47. Fonagy P., et al. "The capacity for understanding mental states: The reflective self in parent and child and its significance for security of attachment". Infant Mental Health Journal 12 (1991): 201-218.

48. Kashdan TB and Rottenberg J. "Psychological flexibility as a fundamental aspect of health". Clinical Psychology Review 30.7 (2010): 865-878.

49. Salvatore S. "Cultural Psychology as the Science of Sensemaking: A Semiotic-cultural Framework for Psychology. In A. Rosa and J. Valsiner (Eds). The Cambridge handbook of sociocultural psychology. Cambridge, UK: Cambridge University Press (2018): 38-47

50. Salvatore S. "Social Life of the Sign: Sensemaking in Society". In J. Valsiner (Ed.), The Oxford Handbook of Culture and Psychology, Oxford, NY: Oxford University Press (2012): 241-254.

51. Wilson A and Weinstein L. "Language, thought, and interiorization A Vygotskian and psychoanalytic perspective". Contemporary Psychoanalysis 26.1 (1990): 24-40.

52. Roisman GI. "The role of adult attachment security in nonromantic, non-attachment-related first interactions between same-sex strangers". Attachment and Human Development 8.4 (2006): 341-352.

53. Pallini S., et al. "The relation of attachment security status to effortful self-regulation: A meta-analysis". Psychological Bulletin 1 Cognitive Neuroscience 2.1 (2018): 55-69. 
54. Allen JG and Fonagy P. "The handbook of mentalization-based treatment". New York, NY: John Wiley and Sons Inc (2006).

55. Midgley N., et al. "Mentalization-based treatment for children: A timelimited approach". Washington, DC: American Psychological Association (2017).

56. Lemma A., et al. "Brief Dynamic Interpersonal Therapy. A Clinician's Guide". Oxford, UK: Oxford University Press

57. McEwen BS. "Stress and hippocampal plasticity". Annual review of neuroscience 22.1 (1990): 105-122.

58. Pakpour AH and Griffiths MD. "The fear of COVID-19 and its role in preventive behaviors". Journal of Concurrent Disorders (2020).

59. Anderson RM., et al. "How will country-based mitigation measures influence the course of the COVID-19 epidemic?" The Lancet 395.10228 (2020): 931-934.

60. Bronfenbrenner U. "The ecology of human development: Experiments by nature and design". Cambridge, MA: Harvard University Press (1979).

\section{Assets from publication with us}

- Prompt Acknowledgement after receiving the article

- Thorough Double blinded peer review

- Rapid Publication

- Issue of Publication Certificate

- High visibility of your Published work

Website: www.actascientific.com/

Submit Article: www.actascientific.com/submission.php

Email us: editor@actascientific.com

Contact us: +919182824667

Citation: Suela Ndoja. "The Components of Puzzle of Existential Fear: An Integrated Neuro-psycho-ecological Model of Understanding Experiences During the Covid-19 Pandemics". Acta Scientific Neurology 5.2 (2022): 11-22. 